\title{
On the Adverse Effects of Glibenclamide Administration
}

\author{
Olga V Akopova* \\ Circulation department, Bogomoletz Institute of Physiology, Ukraine
}

Submission: August 11, 2018; Published: August 24, 2018

*Corresponding author: Olga V Akopova, Circulation department, Bogomoletz Institute of Physiology, NAS of Ukraine, Bogomoletz str. 4, 01601, Kiev, Ukraine; Tel: +38-044-256-24-96; Email: olga.akopova01@mail.ru

\begin{abstract}
Glibenclamide is a widely used drug applied for the blockage of ATP-sensitive K+ channels (KATP channels) ubiquitously present in plasma membranes and mitochondria. While KATP channels opening was shown to be cytoprotective under variety of pathophysiological conditions, in certain cases KATP channel blockage is required for cell survival and the recovery of a living organism. However, in the literature glibenclamide application was shown to produce several adverse effects. In this work some aspects of glibenclamide treatment will be considered in relation to its adverse effects in mitochondria.
\end{abstract}

Keywords: Mitochondria; Glibenclamide; Mitochondrial KATP channels; Bioenergetics; Oxidative phosphorylation

\section{Opinion}

Glibenclamide (glyburide) is a sulfonylurea derivative, N-p[2-(5-Chloro-2-methoxybenzamido) ethyl] benzene sulfonyl$\mathrm{N}^{\prime}$-cyclohexylurea $\left(\mathrm{C}_{23} \mathrm{H}_{28} \mathrm{ClN}_{3} \mathrm{O}_{5} \mathrm{~S}\right)$, which is widely used as a blocker of ATP-sensitive potassium channels (KATP channels) ubiquitously present in plasma membranes and mitochondria. KATP channel is an octameric multiprotein complex that comprises four pore-forming Kir subunits, which are inwardrectifier $\mathrm{K}^{+}$channels, and four sulfonylurea receptors (SUR) [1]. Molecular composition of mitochondrial KATP channel (mKATP) channel remains yet undisclosed and the most part of the notions on the properties of mKATP channel was obtained from the studies on plasmalemmal KATP channels. It is known that glibenclamide binds to SUR subunits to block KATP channel [1,2]. SUR subunit of KATP channel belongs to ATP binding cassette proteins that bind ATP upon its hydrolysis [1-3]. MgATPase activity of SUR subunits is thought to be required for KATP channel functioning, its activation by KATP channels openers and the channel blockage by glibenclamide [3]. The same mechanism is supposed to underlie KATP channel blockage in mitochondria. Generally, no blockage of KATP channels was observed either in the absence of MgATP, or in the presence of $\mathrm{Mg}^{2+}$ or ATP alone [4,5]. Glibenclamide binds to mKATP channels with high affinity, however values of $K_{\mathrm{i}}$ ( 1-6 $\mu \mathrm{M}$ [4]) obtained for mKATP channels were by the order higher than inhibition constants obtained for sKATP channels [1]. This was used by Garlid's group for semiquantitative estimation of the density of mKATP channels in mitochondria from various tissues with fluorescent glibenclamide derivative [6].
Glibenclamide is a widely applied antidiabetic drug, known to stimulate insulin release by blocking sKATP channels in betacells. However, the application of sulfonylureas (glibenclamide and tolbutamide) produced adverse effects in heart and brain, such as increased cardiovascular mortality in patients with diabetes [7], and increased neurodegeneration after hypoxic brain injury [8]. In our work we observed one of the adverse effects of glibenclamide application in vivo caused by the direct effects of this drug on mitochondrial functions.

\section{The impact of glibenclamide treatment on the endurance to physical stress}

It is well known that physical endurance of a living organism to the exercise training requires enough cellular ATP, for the most part produced by oxidative phosphorylation (OxPhos). In our recent studies we examined the impact of mKATP channels opening on the endurance of animals to physical stress. For this purpose, we let the rats swim in a bath with a load and registered swimming time starting from the beginning until exhaustion. The animals were separated in two groups, of which one was treated with glibenclamide intraperitoneally $(1 \mathrm{mg} / \mathrm{kg}$ weight). Based on the ex vivo study, the untreated animals also were separated in two groups: control and those who exhibited elevated mKATP channel activity. As we have shown in our works, glibenclamide administration in vivo was capable of full block of mitochondrial KATP channel, both control and activated $[9,10]$. As we observed by monitoring the swimming time, improved physical endurance 
correlated with elevated mKATP channel activity, whereas the treatment with glibenclamide, and respectively, blockage of mKATP channel, resulted in a dramatic failure of the physical endurance as compared to untreated animals $[9,10]$.

As showed the study of mitochondrial functions in vitro, mKATP channel opening by diazoxide increased phosphorylation efficiency $(\mathrm{P} / \mathrm{O})$, which coincided with improved endurance in animals that exhibited elevated mKATP channel activity in vivo. Meanwhile a fall of $\mathrm{P} / \mathrm{O}$ in the presence of glibenclamide in vitro well agreed with dramatic failure of the endurance, far below the control, under glibenclamide application in vivo, which indicated a correlation between phosphorylation efficiency and physical endurance in such a way that improved efficiency of ATP synthesis coincided with improved endurance to physical stress [10]. However, regardless of the opposite effects on $\mathrm{P} / 0$, dependent on the opening and blockage of mKATP channel, based on the study on isolated mitochondria we concluded that dramatic impact of glibenclamide on physical endurance could not be considered as a reversal of the effects of mKATP channel opening.

\section{The mechanistical background of glibenclamide action in vivo}

To explain dramatic effects of glibenclamide on the physical endurance, the effect of this drug on mitochondrial functions needs to be considered. According to the present knowledge, ATP-sensitive potassium transport, which is directed to the matrix space in energized mitochondria, dissipates $\Delta \mu_{\mathrm{H}}$, a free energy generated by the electron transport chain. Being energy consuming process $\mathrm{K}^{+}$uptake results in obligatory increase in the respiration rate, which in turn reduces membrane potential $\Delta \Psi_{\mathrm{m}^{\prime}}$ dependent on the share of mKATP channel in oxygen consumption. The impact of mKATP channel opening on $\Delta \Psi_{\mathrm{m}}$ is not the same in different cell types. As we have shown earlier, mKATP channel contributed $\sim 30-35 \%$ to the total $\mathrm{K}^{+}$uptake in brain and liver mitochondria. This was of no effect on the membrane potential in rat liver mitochondria, but slightly $(\sim 20 \%)$ depolarized rat brain mitochondria $[11,12]$.

Glibenclamide, as well as the other mKATP channel blocker (5-HD), by blocking ATP-sensitive $\mathrm{K}^{+}$uptake, reversed the effects of mKATP channel opening on respiration and $\Delta \Psi_{\mathrm{m}}$ : reduced the rate of state 4 respiration proportionally to the share of mKATP channel in $\mathrm{K}^{+}$uptake, and restored $\Delta \Psi_{\mathrm{m}}$ in a measure dependent on the share of ATP-sensitive $\mathrm{K}^{+}$transport in oxygen consumption. In the brain, it partially restored ROS production dependent on $\Delta \Psi_{\mathrm{m}}[12]$ and $\mathrm{Ca}^{2+}$ uptake reduced by mKATP channels opening [13]. Thus, glibenclamide restored mitochondrial functions dependent on the rate of ATP-sensitive potassium transport and its share in respiration: state 4 oxygen consumption, $\Delta \Psi_{m^{\prime}}$ part of the ROS production dependent on $\Delta \Psi_{\mathrm{m}^{\prime}}$, and $\mathrm{Ca}^{2+}$ uptake.

However, quite different was with the effect of glibenclamide on oxidative phosphorylation. As we have shown recently [10], neither mKATP channel opening with diazoxide, nor mKATP channel blockage by glibenclamide could be explained as the result of the modulation of mKATP channel conductance.

On Figure 1 the typical effects of mKATP channel opening with diazoxide and the channel blockage with glibenclamide on mitochondrial functions (state 3 and state 4 respiration, respiratory control ratio RCR, the rate of phosphorylation $\mathrm{J}_{\mathrm{ADP}}$ and phosphorylation efficiency P/O) are shown in Figure 1.
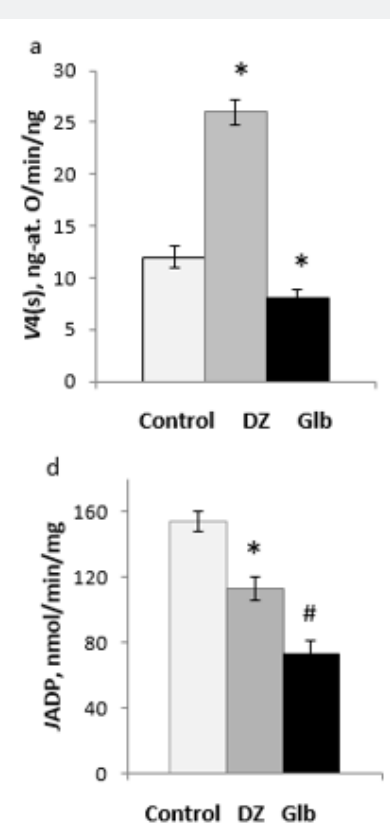
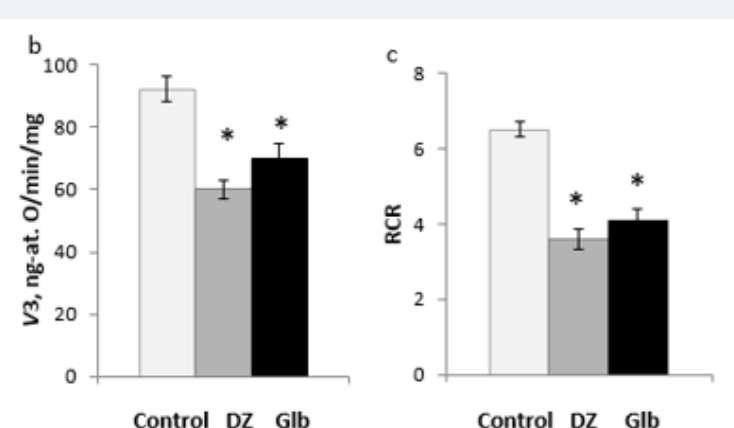

Control DZ Glb

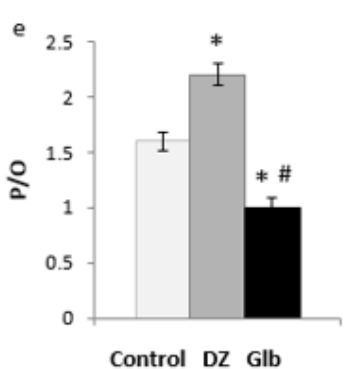

Figure 1: The effects of mKATP channel opener diazoxide (DZ) and blocker, glibenclamide (GIb) on mitochondrial functions: state 4 and state 3 respiration $(a, b)$, respiratory control ratio, RCR (c), the rate of phosphorylation, $\mathrm{J}_{\mathrm{ADP}}(\mathrm{d})$ and phosphorylation efficiency, P/O (e). Incubation medium: $120 \mathrm{mM} \mathrm{KCl}, 0.5 \mathrm{mM}$ EDTA, $5 \mathrm{mM}$ sodium glutamate, $1 \mathrm{mM} \mathrm{KH}_{2} \mathrm{PO}_{4}, 20 \mathrm{mM}$ Tris- $\mathrm{HCl}$ buffer ( $\mathrm{pH} 7.4$ ); ADP was added at $0.2 \mathrm{mM}, \mathrm{DZ}$ at $0.5 \mu \mathrm{M}, \mathrm{Glb}$ at $10 \mu \mathrm{M}$. M $\pm \mathrm{m}, \mathrm{n}=6 .{ }^{*}-\mathrm{p}<0.05$ as compared to control; \# $-\mathrm{p}<0.05$ as compared to diazoxide. 


\section{Open Access Journal of Toxicology}

As show the data, in rat liver mitochondria state 4 respiration was increased by mKATP channel opening proportional to the rate of potassium transport (Figure 1a). mKATP channel opener diazoxide moderately uncoupled mitochondria (reduced RCR), apparently suppressed both the rate of state 3 respiration and the rate of ATP synthesis, but simultaneously increased $\mathrm{P} / 0$ (Figure 1b-1e). Glibenclamide reversed the effect of diazoxide on state 4 respiration (Figure 1a), but similarly suppressed state 3 respiration and decreased the rate of phosphorylation (Figure 1b-1d). However, unlike the case with diazoxide, this was accompanied by the ultimate fall of phosphorylation efficiency (Figure 1e).

Itworth mention that neither suppression of state 3 respiration, nor inhibition of the OxPhos by diazoxide and glibenclamide could be explained by the direct bioenergetic consequences of the modulation of mKATP channel activity. Thus, in the case of diazoxide, similar stimulation of state 4 respiration and mild uncoupling accompanied by the moderate decrease in RCR to the same extent with protonophore could not mimick the effect of mKATP channel opening on the OxPhos (unpublished results). This was not a specific action of diazoxide because similar effects on mitochondrial functions also resulted from the stimulation of $\mathrm{K}^{+}$transport without using any drugs (unpublished observations). Referring to the study on the inhibition of $\mathrm{F}_{0} \mathrm{~F}_{1}$ ATP synthase by $\mathrm{K}^{+} / \mathrm{H}^{+}$ionophore gramicidin [14], we proposed [10] that similar mechanism can underlie OxPhos inhibition by diazoxide, which resulted from the opening of mKATP channel and stimulation of potassium cycling that produced uncoupling of molecular mechanism of $\mathrm{F}_{0} \mathrm{~F}_{1}$ ATP synthase functioning (decoupling).

Unlike its effect on other mitochondrial functions, mKATP channel blocker glibenclamide not only failed to restore OxPhos, but dramatically aggravated the impairment of the OxPhos system caused by mKATP channel opening. It should be stressed, that OxPhos, which is dependent on $\Delta \Psi_{m^{\prime}}$, was suppressed despite mitochondrial energization and restoration of $\Delta \Psi_{\mathrm{m}}$ by glibenclamide. Interestingly, both mKATP channel opening and mKATP channel blockage by glibenclamide resulted in suppression of the OxPhos. However, the effect of glibenclamide on the OxPhos could not be a result of mKATP channel blockage, because the abolition of ATP-sensitive $\mathrm{K}^{+}$transport by varying $\mathrm{K}^{+}$concentration in the medium and reducing $\mathrm{K}^{+}$uptake merely restored control mitochondrial functions.

Considering the mechanism of the blockage of mKATP channel, which requires glibenclamide binding to SUR subunit in the presence of MgATP required for MgATPase activity [3-5], other than mKATP channel, targets of this drug can be suggested to explain its dramatic effect on ATP synthesis. Thus, of the molecular targets of glibenclamide, ability of glibenclamide binding to adenine nucleotide translocase (ANT) already was shown [15]. As showed molecular docking study, glibenclamide binding to ANT can interfere with ADP/ATP translocation [Zym], which in turn should hamper ATP synthesis. Unlike diazoxide, shown to affect several ATP-binding enzymes [16], the ability of glibenclamide to bind to ATP binding proteins was not extensively studied in the literature. However, it is tempting to speculate several targets of glibenclamide among ATP binding proteins, including $\mathrm{F}_{0} \mathrm{~F}_{1}$ ATP synthase, which could be as well one of the putative targets of this drug.

The molecular mechanism in which OxPhos system is affected by glibenclamide needs a more detailed study. Especially important are consequences thereof in vivo. Returning to in vivo experiment, contrary effects of mKATP channel opening and blockage on phosphorylation efficiency correlated well with the opposing effects on physical endurance. However, as showed the results obtained on isolated mitochondria, despite the correlation between $\mathrm{P} / \mathrm{O}$ and physical endurance, dramatic failure of both parameters under the application of glibenclamide was not the consequence of the blockage of mKATP channel activity.

Thus, for understanding of the mechanisms of glibenclamide action in vivo, it remains important to discriminate between the effects ensuing from mKATP channel blockage and the effects not related to potassium transport in mitochondria. As it was shown on isolated mitochondria, glibenclamide, by blocking mKATP channels favors ROS production and mitochondrial $\mathrm{Ca}^{2+}$ overload $[12,13,17]$, which in turn promotes the opening of mitochondrial permeability transition pore [13]. All these effects could contribute to the impairment of physical endurance in vivo. However, from the study of glibenclamide impact on mitochondrial functions, one can hypothesize that the impairment of the OxPhos system, not caused by the blockage of mKATP channels, is the straightest cause for the dramatic failure of physical endurance caused by glibenclamide administration in vivo.

There we considered only one, however important, aspect of glibenclamide impact on mitochondrial bioenergetics and the energy metabolism in a living organism. Regarding glibenclamide impact on mitochondrial functions and the consequences thereof in vivo, there is much remains to be studied yet. Particularly, there is a need to find selective KATP channel blocker, safer for mitochondrial functions. The results of our studies allow for the following conclusion.

\section{Conclusion}

OxPhos system is one of molecular targets of glibenclamide both in vitro and in vivo, which modulation under the action of this drug, as well as the mechanisms underlying coupling between the efficiency of ATP synthesis and physical endurance in a living organism, need to be studied in more details. Dramatic fall of both under the action of glibenclamide was not the result of the blockage of mKATP channel and could not be explained by the effect of glibenclamide on potassium transport or mitochondrial energy state. Plausible explanation of the observed dramatic impact of glibenclamide on the physical endurance in vivo is the impairment of the OxPhos caused by the inhibition of either ANT, or $\mathrm{F}_{0} \mathrm{~F}_{1}$ ATP synthase, which can be considered as one more offtarget adverse effect of glibenclamide, not caused by the direct impact of this mKATP channel blocker on the channel activity. 


\section{References}

1. Foster MN, Coetzee WA (2016) KATP Channels in the Cardiovascular System. Physiol Rev 96(1): 177-252.

2. Moreau C, Prost AL, Dérand R, Vivaudou M (2005) SUR, ABC proteins targeted by KATP channel openers. J Mol Cell Cardiol 38(6): 951-963.

3. Bienengraeber M, Alekseev AE, Abraham MR, Carrasco AJ, Moreau C, et al. (2000) ATPase activity of the sulfonylurea receptor: a catalytic function for the KATP channel complex. FASEB J 14(13): 1943-1952.

4. Jabůrek M, Yarov-Yarovoy V, Paucek P, Garlid KD (1998) Statedependent inhibition of the mitochondrial KATP channel by glyburide and 5-hydroxydecanoate. J Biol Chem 273(22): 13578-13582.

5. Mironova GD, Negoda AE, Marinov BS, Paucek P, Costa ADT, et al. (2004) Functional distinctions between the mitochondrial ATP-dependent $\mathrm{K}^{+}$ Channel (mitoKATP) and its inward rectifier subunit (mitoKIR). J Biol Chem 279(31): 32562-32568.

6. Bajgar R, Seetharaman S, Kowaltowski AJ, Garlid KD, Paucek P (2001) Identification and properties of a novel intracellular (mitochondrial) ATP-sensitive potassium channel in brain. J Biol Chem 276(36): 33369-33374.

7. Brady PA, Terzic A (1998) The sulfonylurea controversy: more questions from the heart. J Am Coll Cardiol 31(5): 950-956.

8. Sun HS, Xu B, Chen W, Xiao A, Turlova E, et al. (2015) Neuronal K(ATP) channels mediate hypoxic preconditioning and reduce subsequent neonatal hypoxic-ischemic brain injury. Exp Neurol 263: 161-171.

9. Akopova O, Nosar V, Gavenauskas B, Bratus L, Kolchinskaya L, et al. (2016) The effect of ATP-dependent potassium uptake on mitochondrial functions under acute hypoxia. J Bioenerg Biomembr 48(1): 67-75.
10. Akopova O, Mankovska I, Nosar V, Gavenauskas B, Kolchinskaya L, et al. (2018) The impact of mitochondrial KATP channel opening on energy metabolism under physical stress. Int J Biochem Physiol 3(1): 000121.

11. Akopova OV, Nosar VI, Bouryi VA, Mankovska IN, Sagach VF (2010) Influence of ATP-dependent $\mathrm{K}(+)$-channel opener on $\mathrm{K}+$-cycle and oxygen consumption in rat liver mitochondria. Biochemistry (Moscow) 75(9): 1139-1147.

12. Akopova OV, Kolchinskaya LI, Nosar VI, Bouryi VA, Mankovska IN, et al. (2014) Effect of potential-dependent potassium uptake on production of reactive oxygen species in rat brain mitochondria. Biochemistry (Mosc) 79(1): 44-53.

13. Skalska J, Debska G, Kunz WS, Szewczyk A (2005) Antidiabetic sulfonylureas activate mitochondrial permeability transition in rat skeletal muscle. Br J Pharmacol 145(6): 785-791.

14. Koeppe RE, Rottenberg H (1989) Mechanism of uncoupling of oxidative phosphorylation by gramicidin. Biochemistry 28(10): 4355-4360.

15. Ziemys A, Toleikis A, Kopustinskiene DM (2006) Molecular modelling of K(ATP) channel blockers-ADP/ATP carrier interactions. Syst Biol (Stevenage) 153(5): 390-393.

16. Dzeja PP, Bast P, Ozcan C, Valverde A, Holmuhamedov EL, et al. (2003) Targeting nucleotide-requiring enzymes: implications for diazoxideinduced cardio protection. Am J Physiol 284(4): H1048-H1056.

17. Kurgaliuk NN, Tkachenko GM (2006) Correction of mitochondrial respiration processes in rats with different resistance to hypoxia under stress by modulators of ATP-sensitive potassium channels. Biomed Khim 52(6): 556-567.

\section{Your next submission with Juniper Publishers will reach you the below assets}

- Quality Editorial service

- Swift Peer Review

- Reprints availability

- E-prints Service

- Manuscript Podcast for convenient understanding

- Global attainment for your research

- Manuscript accessibility in different formats

( Pdf, E-pub, Full Text, Audio)

- Unceasing customer service

Track the below URL for one-step submission https://juniperpublishers.com/online-submission.php 